\title{
Neutron Background Simulations for LEGEND-1000 in a Geant4-based Framework
}

\author{
C.J. Barton* \\ University of South Dakota, \\ 414 E. Clark Street, Vermillion, SD, USA \\ On behalf of the LEGEND collaboration \\ E-mail: C].Barton@coyotes.usd.edu
}

The LEGEND (Large Enriched Germanium Experiment for Neutrinoless double-beta Decay) Collaboration will begin the construction of its initial phase, LEGEND-200, using recently repurposed GERDA infrastructure, with a final 1000-kg installation (LEGEND-1000) in planning. A simulation study of the neutron background is underway, using a custom simulation module based on Geant4. Neutron backgrounds have a strong dependence on laboratory depth, shielding material, and cryostat design. This module has been developed to study cosmogenically-induced neutrons, as well as neutrons from $(\alpha, n)$ reactions. The progress and status of this work will be discussed.

40th International Conference on High Energy physics - ICHEP2020

July 28 - August 6, 2020

Prague, Czech Republic (virtual meeting)

${ }^{*}$ Speaker 


\section{Neutron simulations}

A simulation module using the Geant4[1] software framework was developed primarily for LEGEND-1000[2] simulations, using the "shielding" physics list. It includes custom inputs and a geometry resembling the LEGEND-1000 baseline geometry, shown in Figure 1. The radiogenic neutron flux and energy spectra were calculated using material assay measurements[3] performed for the GERDA experiment as well as the NeuCBOT software package[4]. Cosmogenic muon flux and spectra were based on a parametrization from [5] and detector signal estimates from neutron capture are from [6].
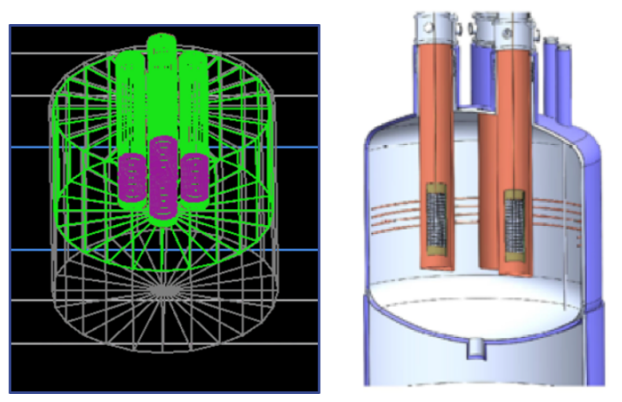

Figure 1: The simulation geometry used in this work (left) and a CAD sketch of the LEGEND1000 baseline design (right).

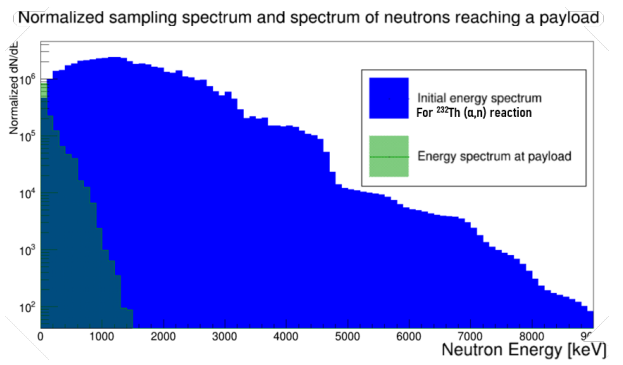

Figure 2: Normalized energy spectra of neutrons from the ${ }^{228} \mathrm{Th}(\alpha, \mathrm{n})$ reaction in the stainless steel cryostat at time of creation (blue) and on contact with a germanium crystal (green overlay).

\section{Radiogenic neutrons}

In the LEGEND-1000 baseline design, some major contributors of radiogenic neutrons are $(\alpha, n)$ reactions and spontaneous fission from impurities in the stainless steel cryostat. The neutron energy spectra are depicted in Fig. 2. The neutrons must propagate through $2 \mathrm{~m}$ of liquid argon at minimum to reach a germanium detector, significantly reducing flux and energy. Neutrons reaching detectors must also be captured in order to generate a signal in the region of interest, decreasing background further. Conservative calculations in this work suggest the background contribution from radiogenic neutrons will be less than $10 \%$ of the total LEGEND-1000 background goal.

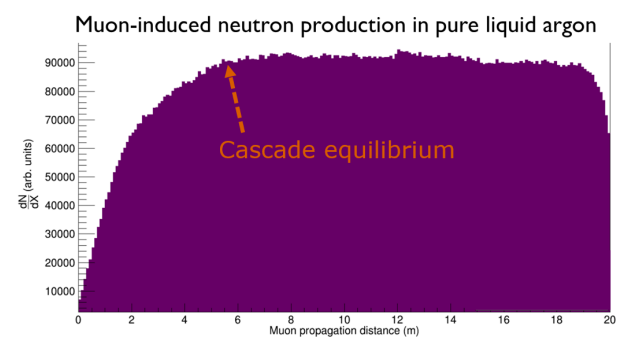

Figure 3: Neutron production rate as a function of muon path length in liquid argon. LEGEND1000 path lengths are in the region of rapid rate increase before equilibrium is reached.

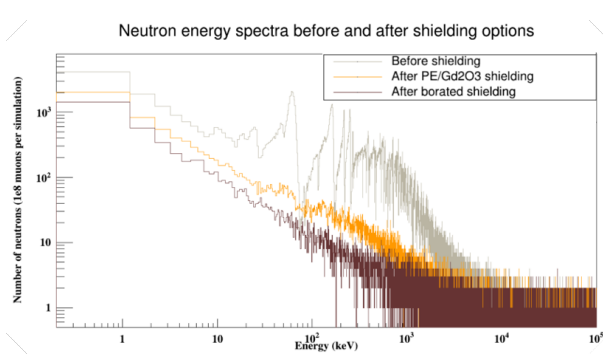

Figure 4: Neutron flux with shielding options described in the text. Shields are $10 \mathrm{~cm}$ thick and cylindrical. 


\section{Cosmogenic neutrons}

Cosmogenically induced neutrons are primarily generated during hadronic showers initiated by cosmic ray muons of $\mathrm{GeV}$ to $\mathrm{TeV}$ energy interacting with nuclei along their trajectories. The resulting particle cascade increases in size until reaching equilibrium. Outside the liquid argon, the water shielding effectively stops muon showers. A key insight of this work was that larger liquid argon shielding could increase cosmogenic neutron flux impinging on the detectors, as shown in Fig. 3. At SNOLab depth and with a minimum argon shielding of 2 meters, the cosmogenic neutron backgrounds are also less than $10 \%$ of the total LEGEND-1000 background goal.

\section{Mitigation strategies}

A solid shield and liquid argon doping are under consideration to mitigate the neutron flux at the detectors. A polyethylene shield with an inner lining of gadolinium oxide and a polyethylene shield doped with a 5\% mass fraction of boron were considered. The shield material can reduce neutron flux at the detectors by up to a factor of two, as in Figure 4, but may introduce new sources of background such as $(a, n)$ reactions in the shields. Liquid argon doping studies are ongoing. Preliminary results suggest that ${ }^{131} \mathrm{Xe}$ doping at various concentrations did not significantly affect neutron flux. ${ }^{3} \mathrm{He}$ doping was effective, but cost and supply considerations may limit procurement.

\section{Acknowledgments}

The author is supported by the National Science Foundation under Grant No. 1812356. This material is based on work supported by the U.S. NSF, DOE-NP, NERSCC and through the LANL LDRD program, the Oak Ridge Leadership Computing Facility; the Russian RFBR, the Canadian NSERC and CFI; the German BMBF, DFG and MPG; the Italian INFN; the Polish NCN and Foundation for Polish Science; and the Swiss SNF; the Sanford Underground Research Facility, and the Laboratori Nazionali del Gran Sasso.

\section{References}

[1] S. Agostinelli et al. Geant4-a simulation toolkit, NIMA 506 (2003) 250.

[2] N. Abgrall et al. The large enriched germanium experiment for neutrinoless double beta decay (LEGEND), AIP Conf. Proc. 1894 (2017) 020027 [physics 1709.01980].

[3] W. Maneschg et al. Measurements of extremely low radioactivity levels in stainless steel for GERDA, NIMA 593 (2008) 448.

[4] S. Westerdale and P.D. Meyers, Radiogenic neutron yield calculations for low-background experiments, NIMA 875 (2017) 57 [ins-det 1702 .02465].

[5] D.-M. Mei and A. Hime, Muon-Induced Background Study for Underground Laboratories, PhysRevD 73 (2006) 053004 [astro-ph 0512125].

[6] C. Wiesinger et al. Virtual depth by active background suppression: Revisiting the cosmic muon induced background of GERDA Phase II, EPJC 78 (2018) 597 [hep-ex 1802 . 05040]. 